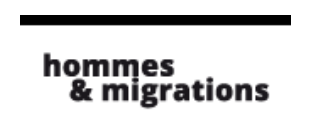

Hommes \& migrations

Revue française de référence sur les dynamiques

migratoires

$1284 \mid 2010$

Migrations et environnement

\title{
Ghana. Migration agricole et usages durables de la terre dans la zone de transition forêt-savane
}

\section{Kees van der Geest}

Traducteur : Françoise Bouillot

\section{OpenEdition}

\section{Journals}

Édition électronique

URL : http://journals.openedition.org/hommesmigrations/1253

DOI : 10.4000/hommesmigrations. 1253

ISSN : 2262-3353

Éditeur

Musée national de l'histoire de l'immigration

Édition imprimée

Date de publication : 1 mars 2010

Pagination : 112-127

ISSN : 1142-852X

Référence électronique

Kees van der Geest, « Ghana. Migration agricole et usages durables de la terre dans la zone de transition forêt-savane », Hommes \& migrations [En ligne], 1284 | 2010, mis en ligne le 29 mai 2013, consulté le 30 avril 2019. URL : http://journals.openedition.org/hommesmigrations/1253 ; DOI : 10.4000/hommesmigrations.1253 
Ghana

\section{Migration agricole et usages durables de la terre dans la zone de transition forêt-savane}

Par Kees van der Geest, géographe, Université d'Amsterdam

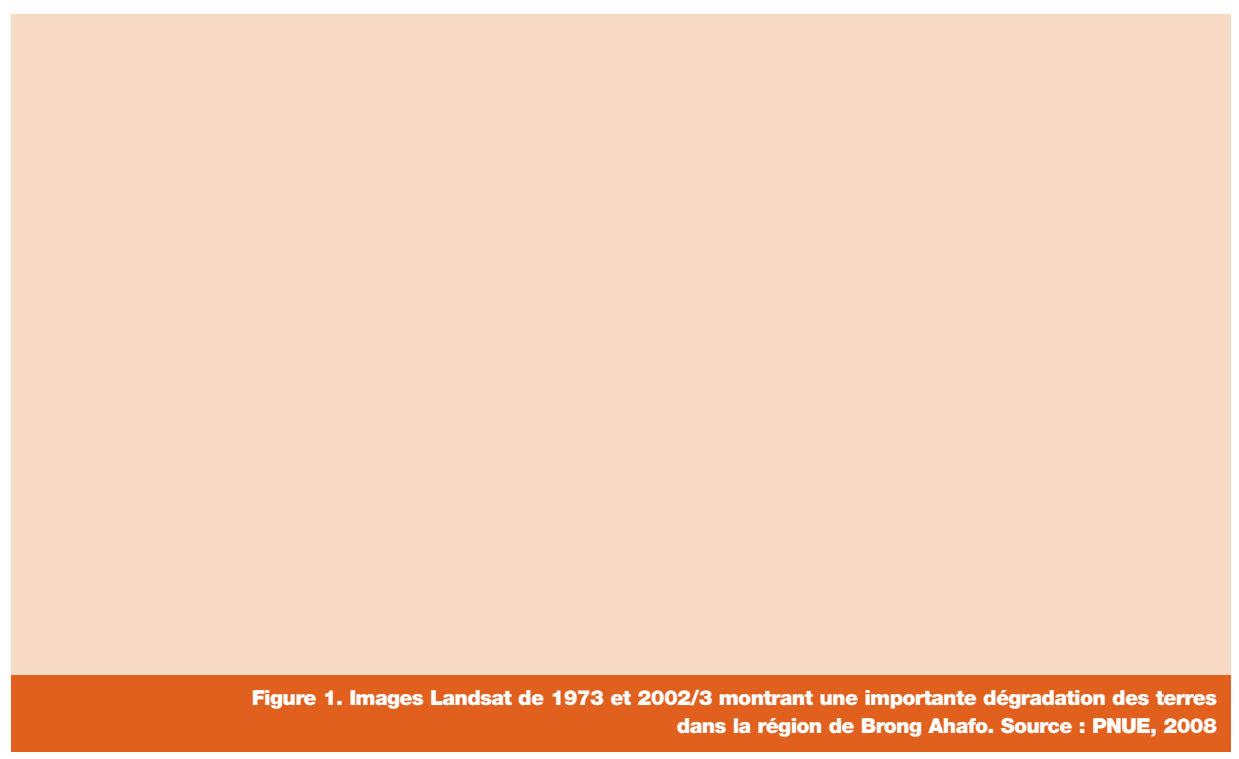

Au Ghana, d'après des images satellites, la zone de transition entre la forêt et la savane a subi en trente ans une profonde dégradation environnementale. La forte immigration de cultivateurs

du Haut Ghana occidental, accentuant une rapide croissance démographique, a accru la pression sur les ressources naturelles. Entre les populations autochtones et les migrants, le partage des terres et les méthodes agricoles demeurent en perpétuel ajustement. Contre les idées reçues qui font des migrants les seuls responsables des pratiques agricoles non durables, l'impact environnemental de l'immigration doit être mesuré sur le terrain 
En 2008, le Programme des Nations unies pour l'environnement (Pnue) a publié un Atlas de l'évolution de notre environnement sur l'Afrique. Cette remarquable collection d'images satellites vise à exposer les changements environnementaux majeurs sur le continent africain. Elle contient notamment deux images montrant la dégradation à grande échelle de la zone de transition forêt-savane au Ghana (voir figure 1 ci-contre). La première image, qui date de 1973, montre une végétation dense, avec quelques parcelles de terre dégradée au nord et autour des grandes agglomérations (en gris clair). Les taches blanches au sud et à l'ouest sont des nuages. Sur la seconde image, prise en 2002/2003, une grande part de la végétation au nord a disparu. La culture sur brûlis et la surexploitation agricole des terres défrichées sont désignées comme les causes principales de cette perte de couverture végétale ${ }^{(1)}$. Mais l'Atlas ne précise pas que la première image a été prise à la fin de la saison des pluies, et la seconde au milieu de la saison sèche. Même si d'autres sources confirment que cette région a connu une déforestation substantielle, la scène aurait paru moins tragique si les deux images avaient été prises à la même saison.

La vision d'une dégradation de l'environnement par les cultivateurs africains qui utiliseraient des méthodes de culture non durables est très répandue, bien que contestée ${ }^{(2)}$. La croissance démographique et la pauvreté sont souvent liées à la déforestation et à la savanisation, puisque l'on estime qu'un nombre croissant de cultivateurs pauvres puise sur la forêt pour pratiquer la culture sur brûlis ${ }^{(3)}$. Selon Carr, s'il existe des preuves réelles d'une corrélation positive entre la croissance de la population et la déforestation au niveau macrotemporel et spatial, elles restent limitées au niveau micro $^{(4)}$. Lambin et al. vont plus loin en affirmant que la démographie comme facteur majeur de la déforestation tropicale est un mythe, ou du moins une simplification ${ }^{(5)}$. Pour les opposants à l'idée que la pauvreté et la démographie sont les premiers facteurs de la dégradation de l'environnement, accuser les pauvres est un legs du passé colonial. Cela tient à l'incapacité des observateurs occidentaux à comprendre les styles de gestion des ressources dans des environnements déséquilibrés ${ }^{(6)}$.

\section{L'impact environnemental des migrants}

La zone présentant des signes de dégradation se situe essentiellement dans la région de Brong Ahafo (voir figure 2). La partie nord de l'image satellite, la plus dégradée, est la première zone de destination des migrants Dagaba ${ }^{(7)}$ issus de la région du Haut Ghana occidental. L'essentiel des terres dégradées se situe dans le district de Wenchi. Cette zone est devenue une importante destination pour les cultivateurs migrants 
dans les années soixante-dix et quatre-vingt. La grande majorité des Dagaba installés dans la région de Brong Ahafo sont de petits cultivateurs qui ont migré en quête de meilleures conditions agro-écologiques ${ }^{(8)}$ Leur zone d'origine au nord du Ghana n'a qu'une seule saison des pluies et les sols sont moins fertiles, surtout dans les parties moins densément peuplées de la région nord-ouest. L'amélioration de la sécurité alimentaire, tant pour les migrants que pour leurs familles restées chez eux, joue un rôle très important dans ce système de migration ${ }^{(9)}$.

En théorie, l'impact environnemental ${ }^{(10)}$ de l'immigration peut avoir deux aspects. D'abord, la migration modifie la taille de la population dans les zones d'origine et de destination. Selon Hugo: "Toutes choses égales par ailleurs (ce qu'elles sont rarement), lémigration va réduire les pressions environnementales à l'origine et les accroître dans la destination ${ }^{(11)}$."

Les études sur la durabilité des méthodes d'exploitation indiquent souvent que les migrants ont des pratiques plus destructrices que les cultivateurs autochtones. Cela s'explique notamment par le fait que la plupart ne possèdent pas la terre qu'ils

Figure 2 : Couverture des images satellites et régions d'origine et de destination des migrants Dagaba au Ghana 
exploitent et considèrent leur séjour comme temporaire, ce qui réduirait leurs motivations à appliquer des méthodes durables pour l'environnement. Ensuite, ils doivent souvent louer la terre, ce qui les encouragerait à "épuiser le sol ${ }^{(12)}$ ". Enfin, on dit souvent que les migrants n'ont pas la même connaissance de l'environnement local que les autochtones, ce qui peut aussi susciter des pratiques non durables ${ }^{(13)}$.

\section{Migration Dagaba et croissance de la population}

La région d'origine des Dagaba est la savane du nord-ouest du Ghana, mais beaucoup ont migré vers le sud. Lors du recensement de 2000, 51 \% des Dagaba vivaient hors de la région du nord-ouest ; 36 \% vivaient dans le sud du Ghana, et 18 \% dans la région de Brong Ahafo, où ils constituaient 6,8\% de la population ${ }^{(14)}$. Outre les migrants Dabaga installés, il existe un mouvement saisonnier de jeunes gens qui profitent de la morte saison au nord pour gagner un peu d'argent comme ouvriers agricoles dans le sud.

Dans la région de Brong Ahafo, les districts de destination les plus fréquents pour les migrants Dagaba se trouvent au nord de la région (voir figure 3). Le district de Wenchi abrite la plus grande proportion de Dagaba, suivi du district voisin de Techiman. Les districts ayant reçu le plus de migrants Dagaba ont connu une dégradation environnementale beaucoup plus forte que les autres. Il s'agit de déterminer si la dégradation est une simple conséquence d'une pression démographique accrue, ou si les cultivateurs migrants ont un impact plus négatif sur la couverture végétale que les autochtones?

Les données des recensements de 1970, 1984 et 2000 montrent que les secteurs nord de la région de Brong Ahafo ont connu dans les dernières décennies une poussée démographique bien plus forte que les régions du sud. Entre 1970 et 2000 - entre les deux images satellite - la population du nord a presque triplé, alors que celle du sud a "seulement" doublée(15).

\section{Procédés d'exploitation et modifications de la couverture végétale}

Amanor \& Pabi ont analysé ces modifications en six endroits du district de Wenchi à partir des images satellites de 1984 et 2000/01 ${ }^{(16)}$. Ils ont trouvé que la "végétation dense" (forêt) s'était réduite sur cinq sites sur six, dans et hors des réserves forestières. 
Figure 3. Immigration Dagaba par district pour la région de Brong Ahafo, Ghana

\section{(a) total ; \\ (b) pourcentage \\ de la population du district ; \\ (c) districts couverts par l'image satellite ; \\ (d) emplacement de la région de Brong Ahafo au Ghana}

Ils soulignent toutefois que cette conversion des terres dans la zone de transition forêt-savane n'est pas un processus linéaire de dégradation, mais plutôt une interaction complexe, dynamique et multidirectionnelle de facteurs humains et naturels. Les sites ayant une agriculture mécanisée et à forte pression externe ont montré le moins de potentiel de regénération, parce que toute la végétation, y compris les souches et les racines, était arrachée. Dans des zones où les systèmes de jachère forestière dominaient, il y avait une forte conversion de forêt en terres agricoles, mais aussi de nombreux changements dans le sens opposé.

Un thème central des études sur l'usage des terres dans la zone de transition est l'impact différentiel des pratiques agricoles des migrants installés (ici "les colons") et 
des autochtones sur la couverture végétale et la fertilité des sols ${ }^{(17)}$. Les autochtones sont souvent présentés comme les "héros" qui préservent la fertilité et la couverture végétale, convertissant même la savane en forêt. Les colons jouent le rôle des "méchants" qui provoquent la déforestation et épuisent le sol. À l'appui de cette histoire de héros, de méchants et de victimes ${ }^{(18)}$, Codjoe soutient que les colons ont envahi les zones "au détriment des peuples indigènes ${ }^{(19) "}$.

L'évaluation très négative des pratiques des migrants peut être liée à une quête de boucs émissaires. Comme nous en avertit Hugo : "Il y a un danger considérable que les migrants impliqués deviennent les boucs émissaires d'un échec général à adopter des politiques durables de la terre et d'autres ressources dans les zones de destination ${ }^{(20)}$."

\section{Méthodologie et lieux de recherche}

Pour étudier la durabilité environnementale des méthodes d'exploitation des migrants, un questionnaire a été distribué dans 203 foyers Dagaba migrants dans huit localités rurales du district de Wenchi et une localité de Techiman ${ }^{(21)}$. Pour permettre une comparaison des méthodes d'exploitation colons/autochtones, un questionnaire similaire a été distribué à 73 foyers de fermiers autochtones dans quatre des neuf villages concernés. Outre les pratiques actuelles, ce questionnaire interrogeait aussi les perceptions de l'usage de la terre "par l'autre" (colons/autochtones et vice versa). Avant la conception des questionnaires et de l'échantillon, une étude pilote de trois semaines a été effectuée dans la région de recherche.

Il est apparu au cours de cette étude préliminaire que la zone avait une écologie extrêmement diverse. Afikorah-Danquah décrit ainsi l'écologie de Wenchi : "La partie sud du district comporte surtout des forêts sèches semi-caduques, alors que le nord consiste en une mosaïque de zones forestières dans des prairies de type savane plus ou moins boisée $e^{(22) " . ~ L e s ~}$ discussions de groupe dans la phase pilote ont aussi révélé d'importantes différences entre villages accessibles et éloignés. Les villages accessibles, situés le long d'une route goudronnée et/ou proches d'un centre urbain (voir figure 4), étaient plus densément peuplés, et la terre arable y était moins facilement disponible. Ces villages étaient des installations anciennes, avec un bon accès aux services : éducation, santé et moyens de commercialisation des produits agricoles. Dans les villages éloignés, les fermiers avaient un meilleur accès aux terres fertiles, mais l'accès aux services était limité, et ils dépendaient de négociants et de propriétaires de camions pour vendre leurs produits. L'écologie diversifiée de la zone de recherche et les histoires divergentes d'installation et de culture suscitent des systèmes différents de gestion environnementale. Pour saisir 
cette diversité, la procédure d'échantillonnage a fait l'objet d'un soin particulier. On a suivi la recommandation de Bilsborrow d'utiliser un échantillon aléatoire pour les foyers, et un échantillon a priori pour le choix des villages ${ }^{(23)}$. On a cherché une représentation égale des foyers migrants dans les trois zones écologiques ainsi que dans les villages accessibles et éloignés (voir tableau 1 et fig. 4). Tous les foyers de migrants de huit villages sur neuf ont été inclus dans l'échantillon. À Krobo, un échantillon au hasard de vingt foyers Dagaba sur un total d'une cinquantaine a été inclu.

\section{Tableau 1 ; foyers Dagara inclus dans Péchantillon $(N=203)$}

\begin{tabular}{|l|l|l|l|l|}
\hline & $\begin{array}{l}\text { Villages } \\
\text { éloignés }\end{array}$ & N & $\begin{array}{c}\text { Villages } \\
\text { accessibles }\end{array}$ & N \\
\hline Savane & $\begin{array}{l}\text { - Fawoman } \\
\text { - Wewa }\end{array}$ & $\begin{array}{l}24 \\
16\end{array}$ & - Subinso No.1 & 29 \\
\hline Transition & - Seikwa & 23 & $\begin{array}{c}\text { - Droboso } \\
\text { - Krobo }\end{array}$ & 23 \\
\hline Forêt & - Adadease & 12 & - Amoahkrom & 34 \\
\hline Total & - Abomfua & 22 & & 106 \\
\hline
\end{tabular}

Figure 4 : Emplacement de la zone d’étude dans la région de Brong Ahafo, Chana 


\section{Les perceptions de l'usage de la terre par "l'autre"}

Dans le questionnaire des foyers, on a demandé aux interviewés d'identifier les différences de méthodes agricoles des migrants et des autochtones. C'était une question ouverte, sans réponses prédéterminées. Il en est résulté d'intéressantes découvertes sur les différences de styles d'exploitation.

Les colons étaient perçus comme ayant des exploitations plus vastes et de meilleures récoltes que les autochtones. L'agriculture est plus souvent l'activité principale des colons, tandis que les autochtones emploient plus de main-d'ceuvre, injectant davantage de capital dans leurs exploitations. Les cultivateurs autochtones sont plus susceptibles d'exploiter leur propre terre et pratiquent davantage la culture de rente (dont l'arboriculture), alors que les colons doivent la louer et pour développer une culture vivrière. Les interviewés précisaient en outre que les colons se servent plutôt de la houe pour préparer la terre, alors que les autochtones préfèrent les coutelas. La durabilité environnementale n'est pas apparue comme un problème dans les perceptions des différences d'exploitation entre colons et autochtones. Ces derniers n'accusaient pas les colons de contribuer à la dégradation des terres par des pratiques agricoles non durables.

\section{Réalités des pratiques et impact sur l'environnement}

Pour déterminer la pression environnementale respective des pratiques agricoles des colons et des autochtones, il faut donc comparer divers aspects de leurs systèmes d'exploitation : régime foncier, taille des exploitations, association des cultures, outils, méthodes de labour, inputs en capital et gestion forestière.

Le tableau 2 donne une information socio-économique basique sur les interviewés. La principale différence entre migrants et colons (colonnes 2 et 3) dans les quatre localités choisies portait sur le niveau d'éducation et l'importance d'un revenu non agricole, plus important chez les autochtones. Dans le groupe des migrants, on notait des différences substantielles entre les zones écologiques (colonnes 4, 5 et 6) et entre les zones accessibles et éloignées (colonnes 7 et 8). Les migrants installés dans des zones de savane tendaient à être plus âgés et avaient migré depuis plus longtemps que ceux de la zone forestière. En outre, les revenus non agricoles des migrants étaient beaucoup plus élevés dans la zone de transition. Cela tient au fait que deux villes rurales relativement importantes (Droboso et Krobo) sont situées dans cette zone. Les 


\section{Tableau 2 : profil socio-économique des migrants par statut,} zone écologique et accès au marché

\begin{tabular}{|c|c|c|c|c|c|c|c|}
\hline & \multicolumn{2}{|c|}{$\begin{array}{l}\text { Statut des migrants } \\
\text { (quatre localités) }\end{array}$} & \multicolumn{3}{|c|}{$\begin{array}{l}\text { Colons par zone } \\
\text { écologique }\end{array}$} & \multicolumn{2}{|c|}{$\begin{array}{c}\text { Colons par accès } \\
\text { au marché }\end{array}$} \\
\hline & $\begin{array}{l}\text { Autochtones } \\
\text { (2) }\end{array}$ & $\begin{array}{c}\text { Colons } \\
\text { (3) }\end{array}$ & $\begin{array}{c}\text { Savane } \\
\text { (4) }\end{array}$ & $\begin{array}{c}\text { Transition } \\
\text { (5) }\end{array}$ & $\begin{array}{l}\text { Forêt } \\
(6)\end{array}$ & $\begin{array}{c}\text { Accessible } \\
\text { (7) }\end{array}$ & $\begin{array}{c}\text { Éloigné } \\
\text { (8) }\end{array}$ \\
\hline Taille famille & 5,5 & 5,8 & 5,8 & 5,8 & 5,4 & 5,4 & 6,0 \\
\hline Femme CF & $11 \%$ & $8 \%$ & $7 \%$ & $14 \%$ & $0 \%$ & $12 \%$ & $1 \%$ \\
\hline Age CF & 49 & 42 & 49 & 43 & 37 & 45 & 41 \\
\hline $\begin{array}{l}\text { Education CF } \\
\text { (années) }\end{array}$ & 5,6 & 2,1 & 2,3 & 2,6 & 2,3 & 3,4 & 1,4 \\
\hline $\begin{array}{l}\text { Revenus } \\
\text { non agricoles } \\
\text { (\%) }\end{array}$ & $84 \%$ & $45 \%$ & $45 \%$ & $52 \%$ & $46 \%$ & $53 \%$ & $41 \%$ \\
\hline $\begin{array}{l}\text { Revenu } \\
\text { non agricole } \\
\text { (c1 000) }\end{array}$ & 3325 & 1516 & 1745 & 2655 & 1615 & 2431 & 1504 \\
\hline Année de migration & & & & & & & \\
\hline - du Haut Ghana & & 1987 & 1980 & 1986 & 1993 & 1984 & 1989 \\
\hline $\begin{array}{l}\text { - dans la résidence } \\
\text { actuelle }\end{array}$ & & 1995 & 1989 & 1993 & 1998 & 1991 & 1996 \\
\hline
\end{tabular}

Note : CF $=$ Chef de famille

possibilités de revenu non agricole y sont meilleures, ce qui explique aussi la forte proportion de femmes chefs de famille. On retrouve la même différence entre villages éloignés et accessibles. En outre, les migrants dans les lieux accessibles avaient en moyenne un meilleur niveau d'éducation que les migrants dans les zones éloignées.

\section{Taille des exploitations et régime foncier}

Comme l'ont souligné d'autres études, le régime foncier de ces groupes varie, ce qui influe sur les prises de décision au plan agricole. Le tableau 3 montre que la majorité des autochtones cultivait leurs propres terres ou "des terres empruntées". Dans le nord-ouest du district de Wenchi, les terres empruntées désignent les champs exploités sous droit d'usufruit. Les migrants paient un loyer annuel au Conseil traditionnel, après quoi ils peuvent exploiter autant de terres qu'ils le veulent. Dans les localités du Sud, la terre empruntée désignait les lopins non loués sur lesquels l'exploitant n'a pas de droits de propriété (par ex. terre de lignage - stool land - ou champs prêtés par des parents ou amis). Dans une zone de savane (Subinso), beaucoup 
de migrants à long terme, qui avaient établi de bonnes relations avec la population autochtone, cultivaient aussi des terres "empruntées".

Dans la zone forestière, le partage des récoltes est le système dominant. L'arrangement le plus courant est le système "abusa" dans lequel le propriétaire prend un tiers de la récolte. Le système du loyer fixe domine dans la zone de transition. Un système relativement nouveau, dit "taungya", est appliqué sur les terres de reforestation de la commission forestière, où les cultivateurs peuvent pratiquer la culture vivrière tant que les arbres sont jeunes ${ }^{(24)}$. Ce système était courant dans un des villages de recherche (Amoahkrom). Sur les 73 chefs de famille autochtones, 18 louaient leurs terres, en général à des agriculteurs Dagaba, sur une base de métayage.

\section{Tableau 3 : régime foncier (en \% de champs)}

\begin{tabular}{|c|c|c|c|c|c|c|c|}
\hline & \multicolumn{2}{|c|}{$\begin{array}{l}\text { Statut des migrants } \\
\text { (quatre localités) }\end{array}$} & \multicolumn{3}{|c|}{$\begin{array}{l}\text { Colons par zone } \\
\text { écologique }\end{array}$} & \multicolumn{2}{|c|}{$\begin{array}{c}\text { Colons par accès } \\
\text { au marché }\end{array}$} \\
\hline & Autochtones & Colons & Savane & Transition & Forêt & Accessible & Éloigné \\
\hline Loyer fixe & 10 & 30 & 11 & 45 & 19 & 42 & 7 \\
\hline Métayage & 1 & 33 & 1 & 37 & 54 & 20 & 52 \\
\hline Stool land & 0 & 27 & 49 & 0 & 0 & 1 & 29 \\
\hline Terres propres & 43 & 0 & 0 & 0 & 0 & 0 & 0 \\
\hline $\begin{array}{l}\text { Terres } \\
\text { "empruntées" }\end{array}$ & 44 & 6 & 38 & 12 & 4 & 21 & 9 \\
\hline Taungya & 2 & 1 & 0 & 2 & 21 & 14 & 0 \\
\hline Autres & 0 & 2 & 0 & 5 & 2 & 2 & 3 \\
\hline Total & 100 & 100 & 100 & 100 & 100 & 100 & 100 \\
\hline
\end{tabular}

Note : Taungya = terres de reforestation de la Commission forestière.

\section{Taille des exploitations et jachère}

Les colons tendent à avoir des exploitations plus vastes que les autochtones, notamment dans les zones éloignées (tableau 4). La grande différence de taille des exploitations des colons entre zones accessibles et éloignées s'explique en partie par le système dominant de métayage. Dans les zones accessibles, les colons paient à l'avance un loyer fixe par acre, alors que dans les zones plus éloignées, le métayage est la façon la plus courante d'avoir accès à de la terre. Pour exploiter une vaste étendue dans les lieux accessibles, les colons doivent avoir une bonne quantité d'argent liquide. En outre, les zones éloignées offrent moins d'opportunités de revenus non agricoles, et les colons se concentrent donc plus sur la culture. 


\section{Tableau 4 : taille des exploitations (en acres)}

\begin{tabular}{|c|c|c|c|c|c|c|c|}
\hline & \multicolumn{2}{|c|}{$\begin{array}{l}\text { Statut des migrants } \\
\text { (quatre localités) }\end{array}$} & \multicolumn{3}{|c|}{$\begin{array}{l}\text { Colons par zone } \\
\text { écologique }\end{array}$} & \multicolumn{2}{|c|}{$\begin{array}{c}\text { Colons par accès } \\
\text { au marché }\end{array}$} \\
\hline & Autochtones & Colons & Savane & Transition & Forêt & Accessible & Eloigné \\
\hline $\begin{array}{l}\text { Taille de } \\
\text { l'exploitation }\end{array}$ & 6,0 & 8,0 & 5,6 & 7,2 & 7,2 & 4,7 & 9 \\
\hline
\end{tabular}

\section{Association des cultures}

La littérature sur les changements d'usage des terres et de couverture végétale dans la zone de transition forêt-savane du Ghana établit une importante distinction entre pratiques agricoles autochtones et modernes. Les formes modernes, intensives et sédentaires de culture exigent de défricher moins de terres, mais la récupération est lente une fois les champs abandonnés. Les cultures modernes typiques sont le maïs (en monoculture), les anacardiers (noix de cajou) et les légumes (destinés à la vente). La noix de cajou et les légumes sont plus répandus chez les autochtones. Le maïs est une importante culture de rente pour les autochtones comme pour les colons, notamment dans les zones de forêt et de transition. Toutefois, les autochtones tendent à cultiver le maïs avec plus d'impacts externes, et plus souvent en monoculture, alors les colons ont recours à la jachère forestière. La culture de l'anacardier est relativement récente dans la zone et, bien qu'elle ajoute à la couverture végétale, elle peut avoir aussi des impacts environnementaux. Il est intéressant de remarquer que les colons se lancent aussi dans l'anacardier, surtout dans la zone de savane où les dispositions du régime foncier sont moins draconiennes. Intégrer des légumes aux cultures est une importante stratégie pour protéger le sol de l'érosion et de l'épuisement, très répandue chez les colons qui utilisent cette pratique dans leur région d'origine. Bien que les colons aient des exploitations plus vastes, leurs ventes de récoltes sont comparables à celles des autochtones.

\section{Outils employés}

Un aspect important de la durabilité de l'usage des terres est le type d'outil utilisé pour défricher et désherber. La plupart des études concluant que les colons venus du nord du Ghana ont des pratiques moins durables critiquent leur usage de la houe, en faveur de l'usage du coutelas par les autochtones. Mais au-delà de ces stéréotypes, aucune différence significative n'a été trouvée dans les trois outils les plus couramment utilisés pour la préparation de la terre - le feu, le coutelas et la houe - par les 
migrants comme les autochtones. En revanche, on note des différences plus marquées dans l'usage de tracteurs pour les labours et de désherbants chimiques. Les deux méthodes sont couramment utilisées par les autochtones, mais certains colons ont aussi adopté ces pratiques. L'usage de tracteurs est associé à une conversion plus permanente de la forêt en prairie. Le désherbant le plus couramment utilisé est le Roundup de Monsanto. Cet herbicide est curieusement promu par le ministère de l'Agriculture du Ghana comme l'alternative environnementale au désherbage manuel. On ignore encore les résultats de ces méthodes en termes de couverture végétale, fertilité des sols, biodiversité et santé humaine.

\section{Techniques de culture}

Le questionnaire a aussi interrogé les pratiques agricoles et les méthodes de labour utilisées par les colons et les autochtones. Le tableau 5 montre qu'une plus forte proportion de colons utilise des méthodes visant à améliorer la fertilité du sol. La seule méthode plus répandue chez les autochtones était l'application de fertilisants non organiques. Ce résultat va à l'encontre de l'idée reçue que les migrants n'investissent pas dans les stratégies de gestion de la fertilité. Les cultivateurs Dagaba dans le Wenchi appliquent des techniques de leur région pour sauvegarder la fertilité des sols. Un phénomène noté par Lambin et al: "Dans certains cas, ces agriculteurs 'déplacés' exacerbent la déforestation par manque de familiarité avec leur nouvel environnement; dans d'autres cas, ils peuvent apporter de nouvelles compétences ayant un impact contraire ${ }^{(25)}$."

\section{Tableau 5 ; techniques de culture (en \% d'agriculteurs)}

\begin{tabular}{|c|c|c|c|c|c|c|c|}
\hline & \multicolumn{2}{|c|}{$\begin{array}{l}\text { Statut des migrants } \\
\text { (quatre localités) }\end{array}$} & \multicolumn{3}{|c|}{$\begin{array}{l}\text { Colons par zone } \\
\text { écologique }\end{array}$} & \multicolumn{2}{|c|}{$\begin{array}{c}\text { Colons par accès } \\
\text { au marché }\end{array}$} \\
\hline & Autochtones & Colons & Savane & Transition & Forêt & Accessible & Éloigné \\
\hline $\begin{array}{l}\text { Association } \\
\text { de cultures }\end{array}$ & 40 & 43 & 60 & 34 & 34 & 42 & 43 \\
\hline $\begin{array}{l}\text { Rotation } \\
\text { des cultures }\end{array}$ & 27 & 46 & 60 & 32 & 41 & 44 & 44 \\
\hline Fertilisants & 21 & 14 & 6 & 19 & 18 & 21 & 6 \\
\hline Fumier animal & 0 & 8 & 7 & 11 & 9 & 12 & 6 \\
\hline Compost & 0 & 1 & 3 & 0 & 0 & 1 & 1 \\
\hline Cover crops & 58 & 64 & 57 & 49 & 68 & 51 & 67 \\
\hline $\begin{array}{l}\text { Méthodes } \\
\text { de conservation } \\
\text { des ressources } \\
\text { (eau/sols) }\end{array}$ & 3 & 32 & 36 & 29 & 8 & 28 & 17 \\
\hline
\end{tabular}




\section{Gestion de la couverture forestière}

Le dernier aspect analysé ici concerne l'impact direct de l'agriculture sur la couverture forestière. On peut penser que pour créer un champ, l'exploitant doit abattre des arbres. Le tableau 6 montre que ce n'est pas toujours le cas. Environ $60 \%$ des colons et $44 \%$ des autochtones indiquaient que la couverture boisée des terres qu'ils exploitaient était assez peu dense pour commencer à cultiver sans abattage. En moyenne, les colons coupaient 87 arbres (11 par acre), contre 162 pour les autochtones, sur des exploitations de six acres au moins, soit 27 arbres par acre. D'un point de vue environnementaliste mainstream, demander aux fermiers combien d'arbres ils ont coupés reviendrait à demander à un voleur combien d'argent il a volé. Mais dans le cadre local, couper des arbres pour cultiver n'est qu'une activité parmi d'autres pour obtenir une bonne récolte. Il n'est pas surprenant que les migrants abattent moins d'arbres que les autochtones. L'abattage est un travail dur et - si paradoxal que cela paraisse aux Occidentaux - il est perçu comme un investissement sur la ferme. Un colon à la situation plus précaire préfere cultiver une terre déjà défrichée. En outre, les autochtones tendent à garder les terres plus vierges et boisées, qu'ils préfèrent cultiver eux-mêmes, tandis que les colons tendent à cultiver les anciennes jachères des autochtones.

Le nombre d'arbres abattus au profit de la culture varie fortement entre zones écologiques, et entre villages accessibles et éloignés. C'est dans la forêt éloignée que les colons abattaient le plus d'arbres. Il s'agit d'une frontière agricole où la plupart des migrants partagent la récolte de maïs dans un système de jachère forestière. Dans les zones de transition plus accessibles (Krobo et Droboso), la principale culture de rente est aussi le maïs, mais c'est une zone d'installation déjà ancienne, riche en terres défrichées et en jachères - ce qui explique qu'il faille y couper moins d'arbres. Les autochtones tendent à couper plus d'arbres, mais ils en plantent aussi davantage. Les trois quarts des interviewés avaient planté, surtout des anacardiers, depuis une dizaine d'années. La taille moyenne des plantations d'arbres des autochtones était

\section{Tableau 6 ; Coupes et reboisement}

\begin{tabular}{|l|c|c|c|c|c|c|c|}
\hline & \multicolumn{2}{|c|}{$\begin{array}{c}\text { Statut des migrants } \\
\text { (quatre localités) }\end{array}$} & \multicolumn{2}{c|}{$\begin{array}{c}\text { Colons par zone } \\
\text { écologique }\end{array}$} & \multicolumn{2}{c|}{$\begin{array}{c}\text { Colons par accès } \\
\text { au marché }\end{array}$} \\
\hline & Autochtones & Colons & Savane & Transition & Forêt & Accessible & Éloigné \\
\hline & $56 \%$ & $41 \%$ & $68 \%$ & $38 \%$ & $44 \%$ & $45 \%$ & $56 \%$ \\
\hline Coupes & 162 & 87 & 102 & 47 & 229 & 39 & 235 \\
Quantité d'arbres & $72 \%$ & $14 \%$ & $32 \%$ & $14 \%$ & $31 \%$ & $33 \%$ & $17,8 \%$ \\
\hline $\begin{array}{l}\text { Arbres plantés } \\
\text { Taille des zones } \\
\text { plantées }\end{array}$ & 2,14 & 0,49 & 0,43 & 0,76 & 0,81 & 0,67 & 0,67 \\
\hline
\end{tabular}


supérieure à deux acres. Il serait intéressant de savoir si les prochaines images satellites indiqueront un effet positif des plantations d'anacardiers sur la couverture végétale. L'enquête a révélé que les colons sont bien plus impliqués dans la plantation d'arbres qu'on ne tend à le penser. Un tiers des colons dans la savane et dans les zones de forêt ont planté des arbres sur leurs exploitations. Dans la zone de forêt, la plantation d'arbres fait partie du contrat, mais les colons de la savane ont commencé à effectuer leurs propres plantations.

\section{Conclusion}

Des études précédentes comparant les pratiques des migrants et des autochtones dans la zone de transition forêt-savane dépeignent des migrants agressifs, aux pratiques destructrices de l'environnement. Les cultivateurs autochtones apparaissent comme des "héros" qui conservent, voire renforcent, la couverture végétale. Mais ces allégations sont peu vérifiées empiriquement, et ne sont pas confirmées par les mesures locales des changements environnementaux. Ces études ont toutes été menées par des chercheurs du sud du Ghana, qui tendent à diaboliser les gens du nord : un danger connu dans les évaluations sur l'impact environnemental de l'immigration ${ }^{(26)}$.

Le questionnaire auprès des colons Dagaba et des cultivateurs autochtones dans les districts de Wenchi et de Techiman a révélé un tableau plus complexe. Il montre d'importantes différences de pratiques, tant entre colons et autochtones qu'entre colons dans des zones écologiques différentes. Les résultats ne confirment pas que les migrants ont des pratiques agricoles plus nuisibles. Bien qu'ils aient des exploitations plus vastes et plantent moins d'arbres que les autochtones, la performance des migrants sur la plupart des autres aspects d'usage durable de la terre était meilleure. Les colons abattent moins d'arbres ; ils utilisent moins de tracteurs et autres éléments associés à une modification de la couverture végétale. Les migrants cultivent plus de patates douces et les autochtones plus de maïs. Les exploitations qui ont introduit la culture des légumes conservent plus d'arbres pour donner de l'ombre. Intégrer des légumes à la variété de cultures est une stratégie efficace de gestion de la fertilité des sols, largement pratiquée par les migrants sur les sols plus pauvres du nord du Ghana. Il en va de même des pratiques de conservation de l'eau, moins courantes chez les autochtones.

Les résultats de cette étude contestent la validité des études précédentes qui accusent les migrants de dégrader les terres dans la zone de transition forêt-savane. L'arrivée de migrants du Haut Ghana occidental a pu accroître la pression sur les terres, mais il n'y a pas de preuves que leurs pratiques agricoles aient un impact environnemental plus négatif que celles des autres cultivateurs. 
Bibliographie

- Abdul-Korah, G. B. "Where is not Home? Dagaba Migrants in the Brong Ahafo Region, 1980 to Present", African Affairs, vol. 106 (422), 2006, pp.71-94.

- Adger, W. N. et al. "Advancing a Political Ecology of Global Environmental Discourses", Development and Change, vol. 32, 2001, pp. 681-715.

- Adjei-Nsiah, S. "Cropping Systems, Land Tenure and Social Diversity in Wenchi, Ghana: Implications for Soil Fertility Management", PhD thesis, Agricultural University of Wageningen, 2006.

- Adjei-Nsiah, S. et al. "Land Tenure and Differential Soil Fertility Management Practices Among Native and Migrant Farmers in Wenchi, Ghana: Implications for Interdisciplinary Action Research", NJAS Wageningen Journal of Life Sciences, vol. 52 (3-4), 2004, pp. 331-348.

- Afikorah-Danquah, S. "Local Resource Management in the Forest-Savanna Transition Zone: The Case of Wenchi District, Ghana", IDS Bulletin, vol. 28 (4), 1997, pp. 36-46.

- Amanor, K. S. et O. Pabi, "Space, Time, Rhetoric and Agricultural Change in the Transition Zone of Ghana", Human Ecology, vol. 35, 2007, pp. 51-67.

- Bilsborrow, R. E. "Sample Design", in Bilsborrow, R. E., A. S. Oberai et G. Standing (dir) Migration Surveys in Low Income Countries: Guidelines for Survey and Questionnaire Design, London \& Sydney: Croom Helm Ltd, 1984, pp. 88-129.

- Carr, D. L. "Proximate Population Factors and Deforestation in Tropical Agricultural Frontiers", Population and Environment, vol. 25 (6), 2004, pp. 585-612.

- Central Bureau of Statistics, Preliminary Report of the 1984 Population Census of Ghana, Accra, Central Bureau of Statistics, 1984.

- Codjoe, S. N. A. "Migrant Versus Indigenous Farmers. An Analysis of Factors Affecting Agricultural Land Use in the Transitional Agro-Ecological Zone of Ghana, 1984-2000", Geografisk Tidsskrift-Danish Journal of Geography, vol. 106 (1), 2006, pp. 103-113.

- De Haan, L. "The Question of Development and Environment in Geography in the Era of Globalisation", GeoJournal, vol. 50, 2000, pp. 359-367.

- Ehrlich, P. R. et A. H. Ehrlich The Population Explosion, New York, Simon \& Schuster, 1990.

- Ghana Statistical Service, Population Data Analysis Reports. Vol 1: Socioeconomic and Demographic Trends Analysis, Accra, Ghana Statistical Service, 2005a.

- Ghana Statistical Service, 2000 Population and Housing Census. Analysis of District Data and Implications for Planning: Brong Ahafo Region, Accra, Ghana Statistical Service, 2005b.

- Hugo, G. "Environmental Concerns and International Migration", International Migration Review, vol. 30 (1), 1996, pp. 105-131.

- Lambin, E. F., et al. "The Causes of Land-Use and Land-Cover Change: Moving Beyond the Myths", Global Environmental Change, vol. 11, 2001, pp. 261-269.

- Leach, M. et J. Fairhead, "Challenging Neo-Malthusian Deforestation Analyses in West Africa's Dynamic Forest Landscapes". Population and Development Review, vol. 26 (1), 2000, pp. 17-43.

- Lentz, C. Ethnicity and the Making of History in Northern Ghana, Edinburgh, University Press, 2006.

- Owusu, V. Migrants, Income and Environment: The Case of Rural Ghana, PhD Thesis Free University of Amsterdam, 2007.

- Richards, P. Indigenous Agricultural Revolution: Ecology and Food Production in West Africa, London, Hutchinson \& Co, (Publishers) Ltd, 1985.

- PNUE, Africa: Atlas of Our Changing Environment, Nairobi, PNUE, 2008.

- Van der Geest, K. "North-South Migration in Ghana: What Role for the Environment?", International Migration, 2010a, à paraître.

- Van der Geest, K. "Migration from Northwest Ghana: Local Perceptions of the Consequences", Africa, vol. 80 (3), 2010b, à paraître.

- Van der Geest, K., A. Vrieling et T. Dietz, "Migration and Environment in Ghana: a Cross-District Analysis of Human Mobility and Vegetation Dynamics", Environment and Urbanization, 2010, À paraître.

- Wardell, D. A., A. Reenberg et C. Tôttrup, "Historical Footprints in Contemporary Land Use Systems: Forest Cover Changes in Savannah Woodlands in the Sudano-Sahelian Zone", Global Environmental Change, vol. 13, 2003, pp. 235-254. 
1. PNUE, Africa: Atlas of Our Changing Environment, Nairobi, PNUE, 2008, p. 185.

2. Wardell et al, "Historical Footprints in Contemporary Land Use Systems: Forest Cover Changes in Savannah Woodlands in the Sudano-Sahelian Zone", Global Environmental Change, vol. 13, 2003, pp. 235-254.

3. S. Afikorah-Danquah, "Local Resource Management in the Forest-Savanna Transition Zone: The Case of Wenchi District, Ghana", IDS Bulletin, vol. 28 (4), 1997, pp. 36-46.

4. D. L. Carr, "Proximate Population Factors and Deforestation in Tropical Agricultural Frontiers", Population and Environment, vol. 25 (6), 2004, p. 586.

5. E. F. Lambin, et al. "The Causes of Land-Use and Land-Cover Change: Moving Beyond the Myths",

Global Environmental Change, vol. 11, 2001, pp. 261-269.

6. P. Richards, Indigenous Agricultural Revolution: Ecology and Food Production in West Africa, London, Hutchinson \& Co, (Publishers) Ltd, 1985 ; M. Leach et J. Fairhead, "Challenging Neo-Malthusian Deforestation Analyses in West Africa's Dynamic Forest Landscapes". Population and Development Review, vol. 26 (1), 2000, pp. $17-43$; L. De Haan, "The Question of Development and Environment in Geography in the Era of Globalisation", GeoJournal, vol. 50, 2000, pp. 359-367.

7. Dans d'autres publications, j'utilise le nom de "Dagara" pour désigner les populations de la région de Nandom, dans le district de Lawra. Ici, le nom "Dagaba" inclut aussi les gens originaires de Jirapa et Nadowli. Voir Lentz (2006) pour une discussion approfondie des identités ethniques dans le Haut Ghana occidental.

8. K. Van der Geest, "North-South Migration in Ghana: What Role for the Environment?", International Migration, 2010a, à paraitre ; G. B. Abdul-Korah, "Where is not Home? Dagaba Migrants in the Brong Ahafo Region, 1980 to Present", African Affairs, vol. 106 (422), 2006, pp. 71-94.

9. K. Van der Geest, "Migration from Northwest Ghana: Local Perceptions of the Consequences", Africa, vol. 80 (3), 2010b, à paraître.

10. Limpact environnemental I est une fonction de la taille de la population (P), de l'affluence (A) et de la technologie (T) Voir P. R. Ehrlich et A. H. Ehrlich The Population Explosion, New York, Simon \& Schuster, 1990.

11. G. Hugo, "Environmental Concerns and International Migration", International Migration Review, vol. 30 (1), 1996, pp. 121.

12. S. Afikorah-Danquah, "Local Resource Management in the Forest-Savanna Transition Zone: The Case of Wenchi District, Ghana", IDS Bulletin, vol. 28 (4), 1997, pp. 36-46 ; S. N. A. Codjoe, "Migrant versus Indigenous Farmers. An Analysis of Factors Affecting Agricultural Land Use in the Transitional Agro-Ecological Zone of Ghana, 1984-2000", Geografisk Tidsskrift-Danish Journal of Geography, vol. 106 (1), 2006, pp. 103-113.

13. E. F. Lambin et al. "The Causes of Land-Use and Land-Cover Change: Moving Beyond the Myths", op. cit.

14. Ghana Statistical Service, Population Data Analysis Reports. Vol 1: Socioeconomic and Demographic Trends Analysis, Accra, Ghana Statistical Service, 2005a.

15. Central Bureau of Statistics, Preliminary Report of the 1984 Population Census of Ghana, Accra, Central Bureau of Statistics, 1984.

16. K. S. Amanor et O. Pabi, "Space, Time, Rhetoric and Agricultural Change in the Transition Zone of Ghana", Human Ecology, vol. 35, 2007, pp. 51-67.

17. S. Adjei-Nsiah, "Cropping systems, land tenure and social diversity in Wenchi, Ghana: Implications for Soil Fertility Management", PhD thesis, Agricultural University of Wageningen, 2006 ; S. Afikorah-Danquah, "Local Resource Management in the Forest-Savanna Transition Zone: The Case of Wenchi District, Ghana", op. cit.; S. N. A. Codjoe, "Migrant versus Indigenous Farmers. An Analysis of Factors Affecting Agricultural Land Use in the Transitional Agro-Ecological Zone of Ghana, 1984-2000", op. cit.

18. W. N. Adger et al. "Advancing a Political Ecology of Global Environmental Discourses", Development and Change, vol. 32, 2001, pp. 681-715.

19. S. N. A. Codjoe, "Migrant versus Indigenous Farmers. An Analysis of Factors Affecting Agricultural Land Use in the Transitional Agro-Ecological Zone of Ghana, 1984-2000", op. cit., p. 103.

20. G. Hugo, "Environmental Concerns and International Migration", op. cit., p. 123.

21. Le travail de terrain s'est déroulé entre septembre 2003 et décembre 2004.

22. S. Afikorah-Danquah, "Local Resource Management in the Forest-Savanna Transition Zone: The Case of Wenchi District, Ghana", IDS Bulletin, vol. 28 (4), 1997, p. 38.

23. R. E. Bilsborrow, "Sample Design", in R. E. Bilsborrow, A. S. Oberai et G. Standing (dir) Migration Surveys in Low Income Countries: Guidelines for Survey and Questionnaire Design, London \& Sydney: Croom Helm Ltd, 1984, pp. 88-129.

24. V. Owusu, Migrants, Income and Environment: The Case of Rural Ghana, PhD Thesis Free University of Amsterdam, 2007, p. 46.

25. E. F. Lambin et al., "The Causes of Land-Use and Land-Cover Change: Moving Beyond the Myths", op. cit.

26. G. Hugo, "Environmental Concerns and International Migration", op. cit. 